\title{
Public Health Decisions Using Point of Care Data from Open Source Systems in Africa
}

\author{
Burke Mamlin ${ }^{\star 1,2}$ and Theresa Cullen 1 \\ ${ }^{1}$ Regenstrief Institute, Indianapolis, IN, USA; ${ }^{2}$ Indiana University, Indianapolis, IN, USA
}

\section{Objective}

We demonstrate an architecture for driving regional public health decisions with automated and semi-automated data collected from open source point of care systems in resource constrained environments.

\section{Introduction}

Ministries of Health in Low and Middle Income Countries (LMIC) are making or trying to make public health decisions for infectious disease conditions like HIV using data garnered from sentinel events and disease tracking in the community. The process of gathering and aggregating data for these case-based reports for is, in all too often a cumbersome or paper-based process. The Center for Disease Control (CDC) was interested in prototyping and piloting approaches that could improve the efficiency and reliability of case reports in resource-constrained environments. One of their primary goals was to demonstrate how electronic data gathered in the front lines of care could be leveraged to automate and improve the reliability of data within case reports driving public health decisions at regional and country levels. OpenMRS is an open source medical record system platform often used in resource constrained environments. ${ }^{1}$ Since OpenMRS is used as an electronic medical record system in several African countries and has been connected to regional or country-level health exchanges, the CDC was interested in building a working solution for electronic case based reporting using OpenMRS and a health information exchange.

\section{Methods}

Working closely with the CDC, we developed a Case-Based Reporting (CBR) module for OpenMRS, using HIV as an initial use case. Trigger events were defined based on sentinel events and key clinical monitoring conditions and these were mapped or added to standard terminologies. We use Health Level 7 (HL7) messaging standards to deliver case reports from OpenMRS to the health information exchange. ${ }^{2}$ We used existing manual workflows and EPI officers to define the needs for a surveillance officer role and the requirements for the CBR module. The module was developed as open source using agile methodologies. OpenHIE (ohie.org) was selected to demonstrate the ability of OpenMRS module to submit an electronic case report to a health information exchange.

\section{Results}

We have a working, open source case-based surveillance module for OpenMRS that comes with nine pre-defined HIV-specific triggers:

- New Case

- New Disease

- New Treatment

- Evidence of Lack of Monitoring

- Evidence of Treatment Failure

- Switched to Second Line Regimen

- Treatment Stopped

- Lost to Follow Up

- Patient Died

We have been able to demonstrate the automatic creation of HIVbased case reports based on data within an electronic medical record system, placement of these proposed case reports into a work queue for a surveillance officer, and successful electronic submission of these case reports into a health information exchange.

\section{Conclusions}

This work demonstrates the ability to develop open source point of care software solutions for LMIC that can be used for sentinel awareness as well as longitudinal monitoring of individual patient care. The current scenarios, trigger identification standards, and messaging specifications are easily accessible and published on the OpenMRS Wiki. ${ }^{3}$ Our incorporation of user centered design through EPI officer engagement helped ensure that our solution is responsive to the end user. The CDC is able to use this solution to demonstrate the feasibility of incorporating electronic case reporting in LMICs and to demonstrate the benefits and promote the adoption of electronic medical record systems and health information exchanges in resource constrained environments. In the next phase of this work, we will be working with the $\mathrm{CDC}$ to identify sites within Africa for deployment and refinement of the CBR module.

\section{Keywords}

Open Source; HIV; Case Reporting; Africa

\section{Acknowledgments}

This work is supported by the Centers for Disease Control and Prevention. Its contents are solely the responsibility of the authors and do not necessarily represent the official views of the Centers for Disease Control and Prevention. We would like to acknowledge the guidance, assistance, and hard work of Eric-Jan Manders, Lisa Murie, Wyclif Luyima, and Jamie Thomas. This work was also made possible through the OpenMRS Infrastructure, which is supported by XSEDE's Jetstream project.

\section{References}

1. Mamlin, B. W., Biondich, P. G., Wolfe, B. A., Fraser, H., Jazayeri, D., Allen, C., et al. (2006). Cooking up an open source EMR for developing countries: OpenMRS - a recipe for successful collaboration. AMIA Annual Symposium Proceedings / AMIA Symposium AMIA Symposium, 529-533.

2. HL7 CDA ${ }^{\circledR}$ R2 Implementation Guide: Public Health Case Report, Release 2 - US Realm - the Electronic Initial Case Report (eICR). Available at http://www.hl7.org/implement/standards/product_brief. cfm?product_id $=436$.

3. OpenMRS documentation on proof of concept for Case Based Reporting. Available at https://wiki.openmrs.org/display/docs/ Case+Based+Reporting+Module.

\section{*Burke Mamlin}

E-mail: bmamlin@iu.edu 